This item was submitted to Loughborough's Research Repository by the author.

Items in Figshare are protected by copyright, with all rights reserved, unless otherwise indicated.

\title{
Towards in-cylinder flow informed engine control strategies using linear stochastic estimation
}

PLEASE CITE THE PUBLISHED VERSION

https://doi.org/10.4271/2019-01-0717

\section{PUBLISHER}

(C) SAE International

\section{VERSION}

AM (Accepted Manuscript)

\section{PUBLISHER STATEMENT}

This paper was accepted for publication in the journal SAE Technical Papers and the definitive published version is available at https://doi.org/10.4271/2019-01-0717

\section{LICENCE}

CC BY-NC-ND 4.0

\section{REPOSITORY RECORD}

Butcher, Daniel, and Adrian Spencer. 2019. "Towards In-cylinder Flow Informed Engine Control Strategies Using Linear Stochastic Estimation”. figshare. https://hdl.handle.net/2134/36690. 


\title{
Towards in-cylinder flow informed engine control strategies using Linear Stochastic Estimation
}

\author{
Author, co-author (Do NOT enter this information. It will be pulled from participant tab in \\ MyTechZone) \\ Affiliation (Do NOT enter this information. It will be pulled from participant tab in MyTechZone)
}

\begin{abstract}
Many modern I.C. engines rely on some form of active control of injection, timing and/or ignition timing to help combat tailpipe out emissions, increase the fuel economy and improve engine drivability. However, development of these strategies is often optimised to suit the average cycle at each condition; an assumption that can lead to sub-optimal performance, especially an increase in particulate (PN) emissions as I.C. engine operation, and in-particular its charge motion is subject to cycle-to-cycle variation (CCV). Literature shows that the locations of otherwise repeatable large-scale flow structures may vary by as much $25 \%$ of the bore dimension; this could have an impact on fuel break-up and distribution and therefore subsequent combustion performance and emissions. In the presented work, a method is presented that allows full-field flow velocity information to be estimated in real-time from only a limited number of point velocity measurements using linear stochastic estimation (LSE). Three sensor arrangements - single bisecting 'line-of-sight', a central cluster and a circumferential ring - which are deemed applicable to implementation in an I.C. engine are compared over all test flow conditions, with all providing useful estimations of the flow field. It is shown how with even a modest number of point measurements it is possible to achieve at least $85 \%$ correlation between estimates and original data allowing cycle characterisation to be achieved. Information gathered from this technique could provide inputs to engine control strategies to account for the CCV of the in-cylinder flow.
\end{abstract}

\section{Introduction}

In order to further reduce emissions and improve fuel economy and engine drivability, almost all modern I.C. engine rely on some form of active control for valve timing, injection strategy and/or ignition timing. Developing strategies, for example for fuel injection, in a Gasoline Direct Injection (GDI) engine requires some knowledge (assumed or otherwise) of how the in-cylinder flow will behave around injection timing particularly when considering particulate emissions $(\mathrm{PN})$.

While the trend in gasoline engines has been away from Port Fuel Injection (PFI) towards GDI; the latter have been shown to emit more PN emissions [1]. A study by Johansson et. al. [2] found a link between local AFR (i.e. stratified charge) and an increase in the formation of PN emissions. This is due to the reduction in soot oxidation in richer regions that exist in stratified injection strategies

Page 1 of 11
$[3,4]$. However, it has been found that even under homogenous strategies, these increases in PN emissions are still evident due to imperfect mixing and air-fuel distribution[5]. The study found that injection operating parameters, particularly injection pressure can influence the PN emissions suggesting it is a suitable control variable.

In the case of spray-guided and air-guided approaches, the strategy relies on the in-cylinder motion to break-up and distribute the fuel. However, I.C. engines are subject to cycle-to-cycle variation (CCV) which means any assumed air flow structures will not necessarily exist in every cycle - and therefore potentially leading to increased $\mathrm{CCV}$ of combustion performance. Despite CCV being the focus of much research over the years, for example[6-10] to name but a few, there is still understanding to be gained. An approach suggested here is to instead make measurements in real-time on the engine that allow control strategies to be adjusted for these variations in flow. This may either feed into existing active systems such as injection timing or pressure or novel developing techniques such as vortex generating jets which aim to control large scale structures within the I.C. engine [11].

In the presented work, a method for determining an estimate of the in-cylinder flow characteristics is presented. Linear stochastic estimation (LSE) makes use of a few measurement points and correlation statistics to predict the full velocity field. The estimated velocity fields may then have subsequent calculations such as identification of vortex center location/s. The approach taken uses emulated velocity point measurements set-up in three schemes that could realistically be implemented in a production I.C. engine and assesses the representativeness of the estimations. For the purposes of the presented development, full field, planar velocity data acquired by PIV in an optical engine is used to allow various selection of emulated sensor points and also the original flow field to be known for comparison. The PIV data is used for both the velocity fields and to provide virtual 'sensor' measurements in this case.

The technique is applied to the flow under a range of engine operating conditions from literature [12], achieved using asymmetric VVT strategies. Thus providing a wide range of structures which could potentially be present in real I.C. engine operation.

\section{Linear stochastic estimation}

LSE is a technique allowing estimation of a quantity given measurement of another quantity and the correlation between them. It 
was first introduced to the field of turbulent flows during the 1970's by Adrian $[13,14]$ to approximate conditional averages of turbulent flow. Since, many research groups have utilised the methodology in a range of applications in the field. For example, Cole et. al. [15] showed how the technique could be used with hot-wire anemometers to estimate a coarse velocity grid. Whilst the work presented here considers only LSE, it is worth discussing that LSE fits in a set of techniques including higher order estimation such as quadratic stochastic estimation (QSE), which is more applicable when correlating acoustic pressure as shown by Druault et. al. [16].

The application of LSE is a two-step process. Firstly, a correlation matrix $(a)$ between a sensor value, $p$ and each point in the vector field $u$ is calculated. For this part, simultaneous measurements of both the velocity field and the sensor are required as shown in Equation 1 at a common time, $t$. The dimensions of the $a$ matrix are equal to the number of velocity points $\mathrm{x}$ the number of sensor locations (r).

$\left\langle u(x, t) p_{q}(t)\right\rangle=\sum_{r=1}^{N_{R}} a_{r}(x)\left\langle p_{r}(t) p_{q}(t)\right\rangle$

$u_{e s t}(x, t)=\sum_{r=1}^{N_{R}} a_{r}(x) p_{r}(t)$

Following the calculation of the $a$ matrix, it may be used to estimate the velocity field, given only the sensor scalars by linear combination as in Equation 2. Therefore once the $a$ matrix has been calculated for a given application, only the sensor scalars must be known at time $t$ in order to estimate the instantaneous velocity field, $u_{\text {est }}(x, t)$.

In turbulent flows, it is common for the LSE technique to be combined with proper orthogonal decomposition (POD). The POD technique developed by Lumley et. al. [17] (also known as KarhunenLoève decomposition or principle component analysis) is shown to effectively separate large-scale coherent flows and small scale turbulence[12,18-21]. For this reason it has often been used for the investigation of cyclic variation in the internal combustion engine $[6,18,19,22,23]$.

In much of the existing literature, the sensors used as inputs to the estimations are wall pressure sensors [24,25] or microphone arrays [26]. As suggested already, this requires a strong cross-correlation between then quantities and the velocity field. Arnault et. al. [27] describes the limitations of using such approaches when attempting to estimate the smaller scales of turbulence. However, the work agrees with that by Muradore et. al. [28] and Cohen et. al. [29] that the accuracy of estimations may be improved by optimisation of sensor location, finding that by placing the sensors close to the POD spatial mode maxima accuracy is improved. However, it is worth being mindful of the suggested application to I.C. engines, where the sensor location may be somewhat restricted.

Work by Kerhervè et. al. [30] shows how it is possible to take velocity measurements as sensor inputs to estimation. This is particularly useful for example, on that work when 'sensor' velocity information is available at a high temporal resolution and combined with high spatial resolution measurements using the POD-LSE technique.

Due to the higher levels of spatial correlation, point velocity components are expected to provide a highly effective input to the estimations and are therefore considered in the presented work. In addition, recent developments in line of sight techniques suited to velocity measurement, such as coaxial volumetric velocimetry introduced by Schneiders et. al. [31] provide promise in delivering more practical application in I.C. engines.

\section{In-cylinder velocity flow fields}

Whilst the presented paper focuses on a methodology for the estimation of flow taxonomy, it was necessary to utilise sets of incylinder flow data for investigation and validation. Further, those velocity fields should have significantly differing features to effectively validate the robustness of the presented technique.

The velocity fields used throughout the presented work were acquired using the particle image velocimetry (PIV) technique in the Lotus SCORE. The experimental data has previously been presented [12] with detailed analysis of the flow fields and the impact of asymmetric valve timing strategy detailed, however a summary of the test conditions and acquisition parameters are given in this section.

\section{Experimental set-up and conditions}

All experiments were carried out in a single cylinder optical research engine, the Lotus SCORE (Figure 1). The engine is capable of fired or motored operation up to 5000 RPM and features fully variable valve timing (lift and phase) independently on all four valves via the Lotus Active Valve Train (AVT) system.

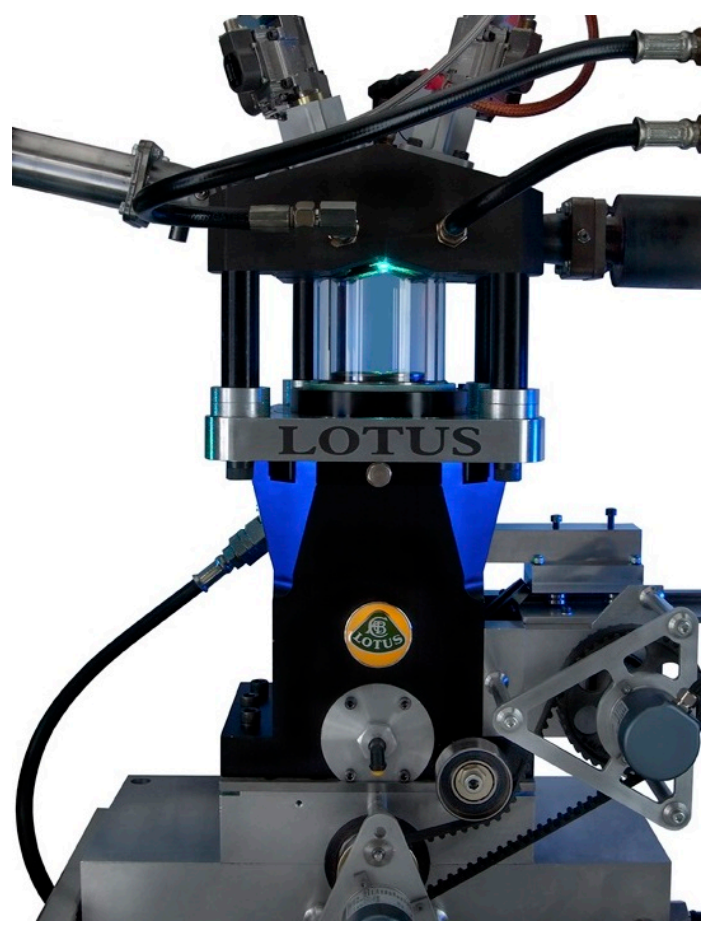

Figure 1. Lotus SCORE - optical research engine

SCORE is a pent-roof, flat piston type engine with the main specifications detailed in Table 1 . The exhaust valve strategy used a polynomial curve fit based on the timings and lift presented in Table 1. In order to generate a range of in-cylinder flow structures, the AVT system was used to generate asymmetric lift profiles on the intake valves.

Page 2 of 11

10/19/2016 


\begin{tabular}{|l|l|}
\hline Swept volume & $0.5 \mathrm{~L}$ \\
\hline Stroke & $88.0 \mathrm{~mm}$ \\
\hline Bore & $82.1 \mathrm{~mm}$ \\
\hline Compression ratio & $10: 1$ \\
\hline Intake / exhaust valve diameters & $31 / 26 \mathrm{~mm}$ \\
\hline Intake open / close / max. lift & $-15^{\circ} / 225^{\circ} / 9.35 \mathrm{~mm}$ \\
\hline Exhaust open / close / max. lift & $495^{\circ} / 15^{\circ} / 9.35 \mathrm{~mm}$ \\
\hline Piston window diameter (available) & $60 \mathrm{~mm}(52 \mathrm{~mm})$ \\
\hline
\end{tabular}

The prescribed valve open and close times were maintained, however for the maximum valve lift, this was scaled as a percentage for one valve only, whilst the other intake valve maintained the maximum lift specified. Three scaling factors, referred to maximum valve lifts (MVL) are considered for the presented work: full lift (100\%), 40\% and $20 \%$. Intake lift profiles for the variable valve are graphically depicted in Figure 2.

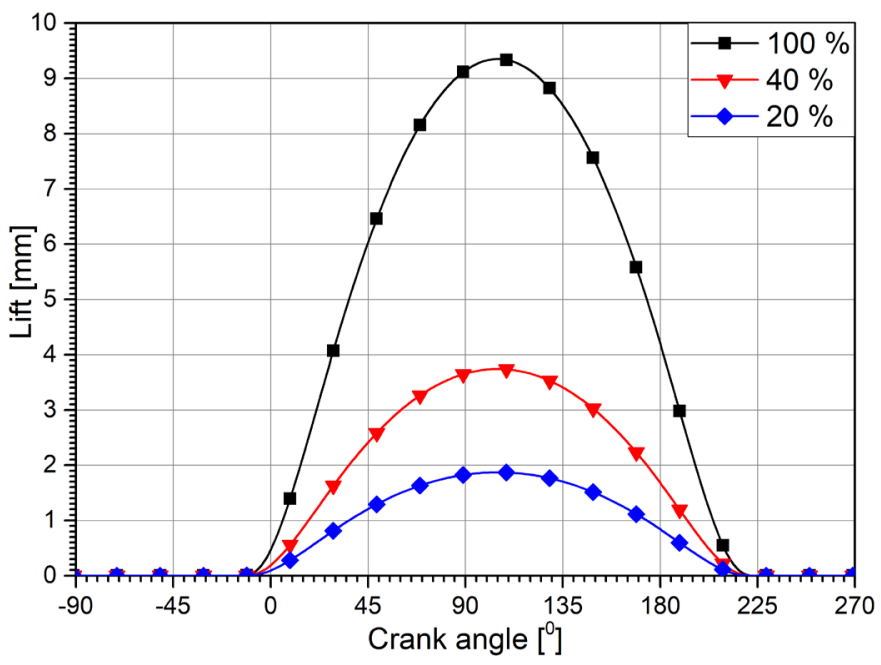

Figure 2. Variable intake valve lift strategies

For all tested valve strategies, the engine speed was set to 2000 RPM and manifold pressure was nominally held at 400 mbar (i.e. part load condition). Whilst it would be possible to extend the operating conditions, it was deemed not necessary. This is because the intention of the experimental campaign was to provide a range of different flow structures, which is shown in literature to be achievable with the presented strategies[12]. In addition, it is further shown in literature that similar flow structures should be present over all realistic engine speeds, simply scaling with speed [32]. Therefore, all data was collected at $75^{\circ}$ crank angle (intake stroke) with differences in acquired flow fields only as a result of the valve strategy.

\section{Velocity field acquisition}

All PIV data was acquired and processed using a LaVision FlowMaster system. This comprised of a light-source: New Wave Solo Nd:YAG pulsed dual cavity laser operated at the second harmonic frequency (532 nm, green) and associated optics, a LaVision FlowMaster 3S 1MP camera fitted with a $60 \mathrm{~mm}$ Nikkor macro lens, a LaVision timing unit (PTU9) and the LaVision DaVis

Page 3 of 11

v8.2 software. The system was synchronised to the engine using a crank-shaft mounted optical encoder; driven on a 2:1 gear to simulate a camshaft and therefore provide $720^{\circ}$ timing.

PIV seeding particles are introduced directly into the intake manifold, well upstream of the intake valves to ensure a sufficiently homogeneously prepared flow. A LaVision aerosol generator is fed with olive oil (density $900 \mathrm{~kg} / \mathrm{m}^{3}$ ) to provide a mist of $1 \mu \mathrm{m}$ particles. Literature suggests this is suitable for application to in-cylinder flows. Melling shows the particles are capable of following up to $10 \mathrm{kHz}$ fluctuations [33].

The laser is shaped by $-20 \mathrm{~mm}$ optics to deliver a uniform light sheet covering the cylinder bore at a height $25 \mathrm{~mm}$ below the bottom of the pent roof (depicted in Figure 3). Delivering the sheet in this manner ensured the measurement area (i.e. that visible through the piston window) was uniformly and sufficiently lit. The height of the sheet was set to ensure there was adequate distance to minimise valve glare whilst allowing measurement during the intake stroke, i.e. the sheet should be well above the piston height at the time of measurement.

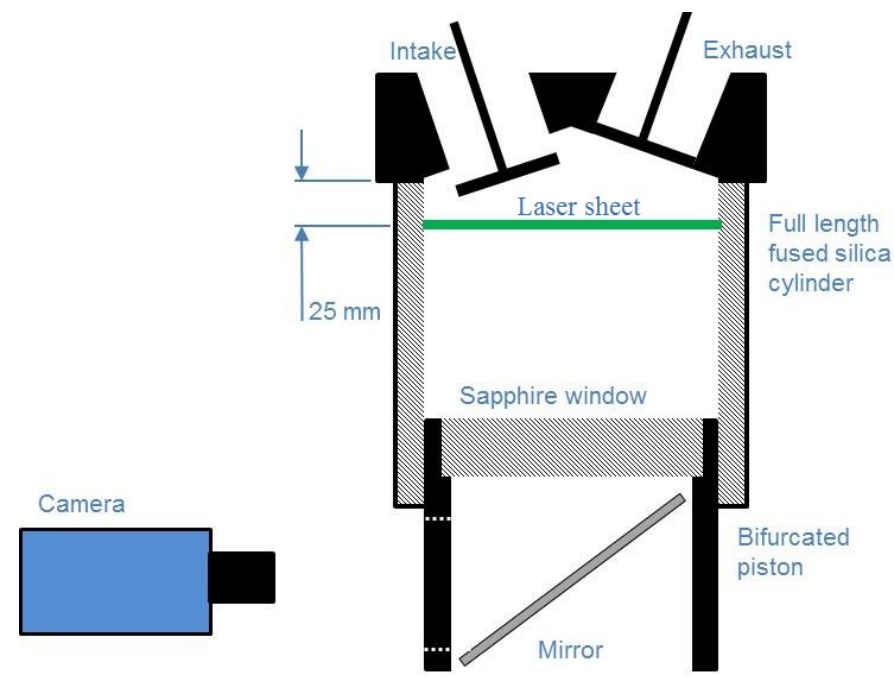

Figure 3. Experimental schematic

The camera was positioned to view the swirl (horizontal) plane through the sapphire piston window. This was achieved using a $45^{\circ}$ mirror placed inside the Bowditch piston arrangement. The selected lens provides a field of view of $86 \times 69 \mathrm{~mm}$. Given the sensor resolution of $1280 \times 1024$ pixels, the physical resolution can be calculated as $69 \mu \mathrm{m} /$ pixel. The f-number was set to 11 , resulting in a particle image diameter of 2.3 pixels calculated according to Arian and Westerweel [34]. This was sufficient to ensure there was no peak-locking, which was subsequently confirmed during initial set-up tests within the DaVis software.

Image pre-processing and vector field calculation was carried out in the $\mathrm{DaV}$ is software. A single pre-processing step of sliding minimum background correction was carried out. This was found to be the most suitable technique in this this application as it effectively accounts for the accumulation of fouling of the optical surfaces, both the liner affecting the delivered light intensity uniformity and the piston sapphire window. The latter was particularly affected by impingement of oil particles, a further factor in selecting the light sheet height discussed earlier. Multi-pass, reducing size interrogation regions are used for vector computation. An initial pass of $64 \times 64$ with $50 \%$ overlap is performed, followed by two further passes at 32 
x 32 with $87 \%$ overlap. Finally, vector fields were down-sampled to a physical grid of $1.1 \times 1.1 \mathrm{~mm}$ to reduce the computation effort requirement for the presented study. Literature suggests that the expected integral length scale should be approximately $4 \mathrm{~mm}$ [35-37] and therefore this down-sampling should not detract from conclusions drawn from this work. For orientation, a typical velocity field is presented in Figure 4, where the approximate locations of valves and the spark plug are indicated.

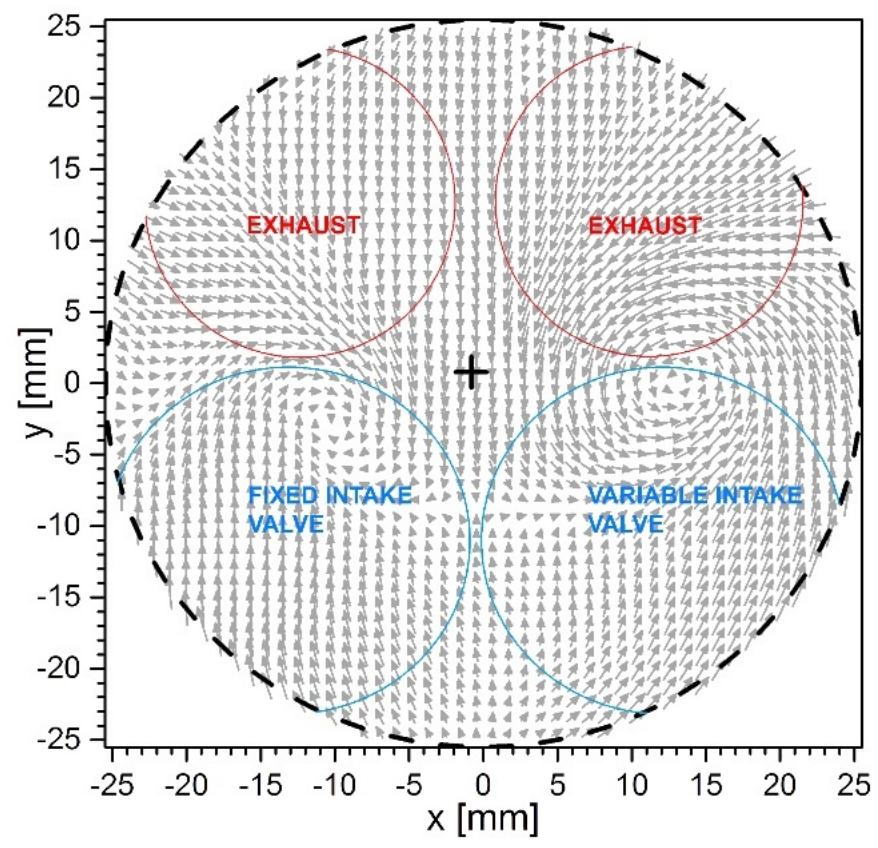

Figure 4. Field of view with indicative valve and spark plug locations

For each tested condition, a total of 800 instantaneous image pairs and therefore velocity fields - were acquired at $75^{\circ} \mathrm{CAD}$. This timing was chosen as it is close to maximum valve lift and therefore maximum in-cylinder flow velocities - maximising the difference in flow structures.

\section{Ensemble average flow fields}

As previously mentioned, 800 velocity fields were captured in each condition. To aid in visualisation, the ensemble average has been computed for each MVL condition and presented in Figure 5.

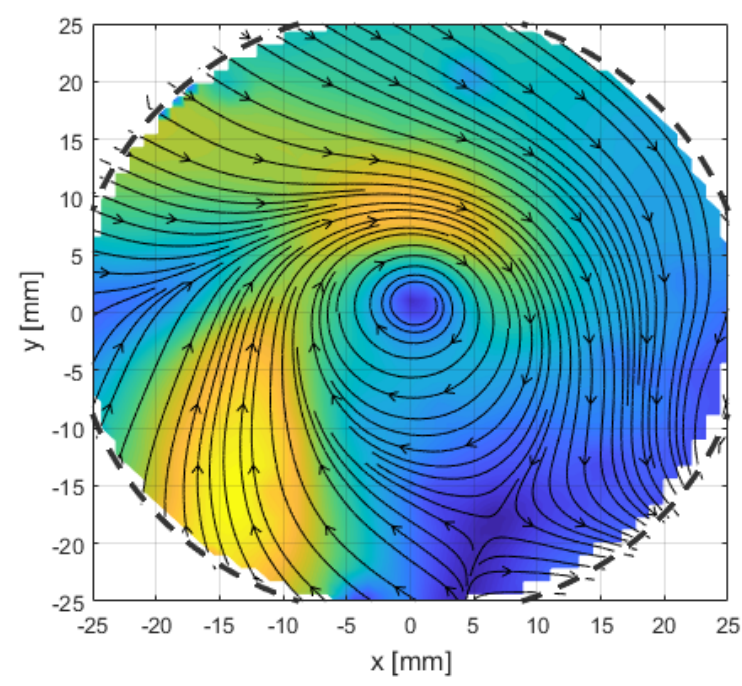

(a) $20 \% \mathrm{MVL}$ 


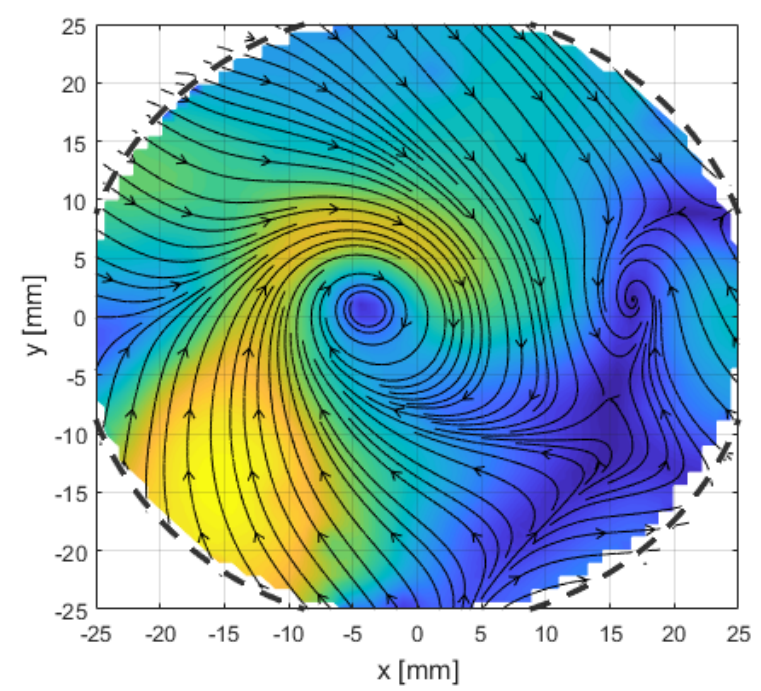

(b) $40 \% \mathrm{MVL}$

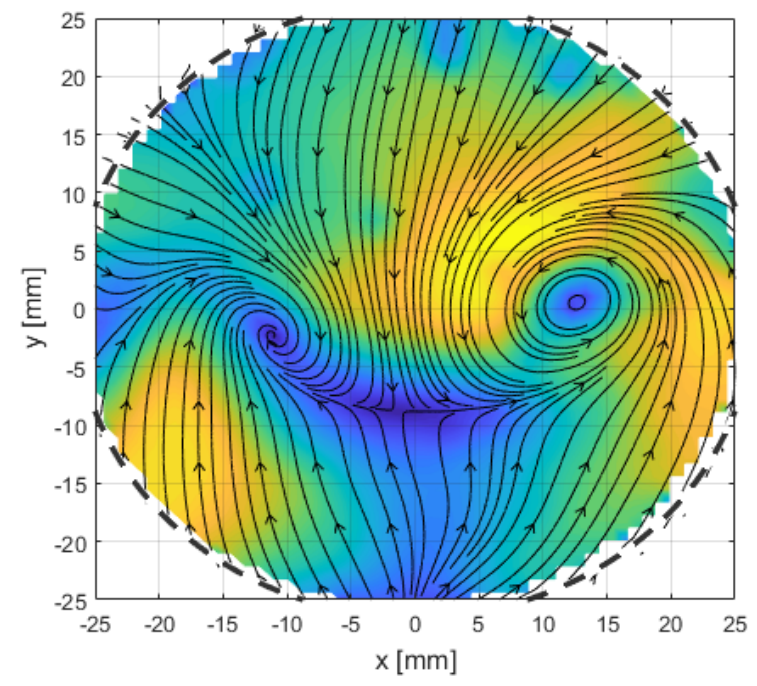

(c) $100 \% \mathrm{MVL}$

Figure 5. Ensemble average flow fields for the three MVL conditions

Examining the three conditions presented in Figure 5, each has distinctive characteristics. The 20\% MVL condition (Figure 5(a)) exhibits a large, single vortex structure which is approximately centrally located within the cylinder. The $100 \%$ MVL condition (Figure 5(c)) exhibits a counter-rotating vortex pair, which is more commonly reported in literature for IC in-cylinder flow. The pair is approximately centrally located, with a bias towards the positive xdirection; attributed to a slight asymmetry in the feed to the intake runners. The flow structures in Figure 5(b), the 40\% MVL condition, are ill-defined by the ensemble average. Earlier work has identified high cyclical variability of the spatial location of the vortex located in the positive $\mathrm{x}$-direction. Further, the presence of this vortex varies [12] - i.e. some cycles are single vortex style while others feature a pair, similar to $20 \%$ MVL and $100 \%$ MVL respectively. This phenomena is demonstrated in Figure 6, where an example of both the single vortex structure and counter-rotating vortex structure are depicted. In both of these cases, the vortex located in the negative $\mathrm{x}$ direction is clearly a strong vortex, with a relatively high local velocity magnitude associated. However, in the case of Figure 6(a) there is arguably some evidence of a vortex forming towards the positive $\mathrm{x}$-direction, but with almost negligible flow velocity, showing evidence of relatively stagnant flow, at least during the time of acquisition $\left(75^{\circ} \mathrm{CAD}\right)$. This could suggest that the formation of this secondary vortex is temporally unstable and its existence is subject to cyclic variation.

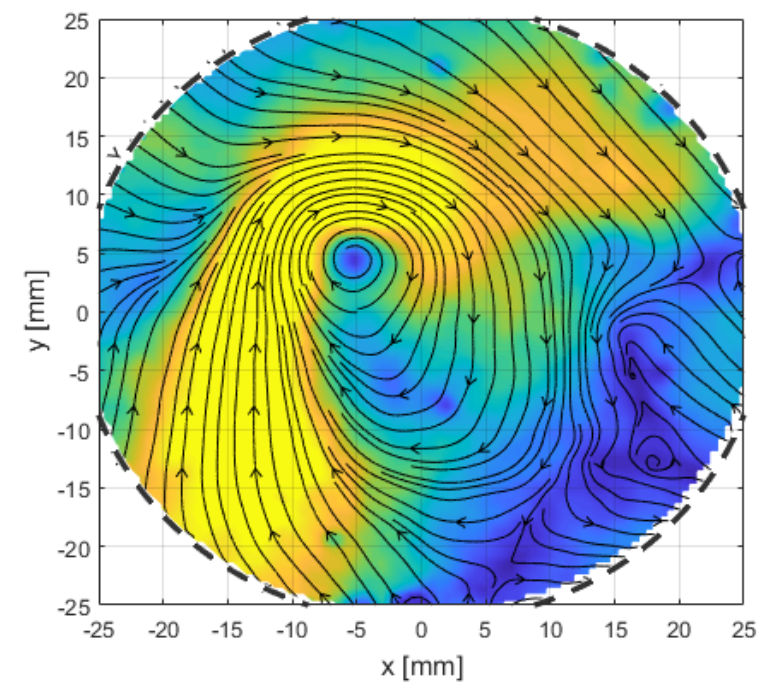

(a) Single vortex example

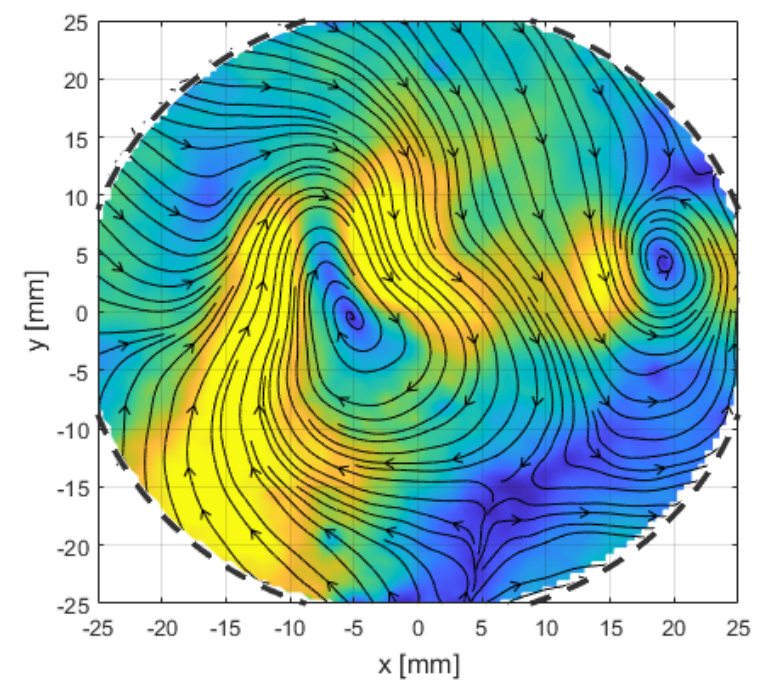

(b) Vortex pair example

Figure 6. Examples of instantaneous cycles in 40\% MVL condition

Engine design may rely on these large in-cylinder flow structures to both break down the fuel by promoting increased flow velocity and therefore turbulence intensity and for the convective transport of the fuel. This bi-modal switching mechanism presents a problem for developing an effective flow guided injection strategy. Therefore, it should be the aim of the applied LSE sensor system to effectively categorise the current cycle flow condition. As it is not practical for an on-line control system to make use of a highly spatially dense data-set, discrete categorisation of flow scheme i.e. number of vortices and/or location and strength could be beneficial as inputs to an adaptive injection strategy. It would be possible to calculate either of these descriptors from resulting estimations using this technique.

Page 5 of 11 


\section{Application of LSE to in-cylinder velocity fields}

\section{LSE using velocity components as scalar sensors}

Literature discussed in the introduction primarily considered sensor inputs to LSE applications, namely pressure and/or microphone based sensing arrays. In practical application of the estimation methodology there is little difference using velocity measurements, as each velocity component, in this case $u_{\mathrm{x}}$ and $\mathrm{u}_{\mathrm{y}}$ may be treated separately as scalars. In this application, the estimation is carried out twice, once for each component. Butcher and Spencer[38] show how by using the appropriate velocity component a higher level of correlation is observed leading to fewer sensors required. Note, it would be possible to estimate both of the velocity components using only a single input scalar sensor, however, this would likely require more sensors i.e. more measurement locations due to the weaker crosscorrelation.

As LSE requires both the velocity field and the sensor, in this case sensor velocity component, to be known simultaneously for the generation of the $a$ matrix, the same data-set may be used for both. As shown in [38], the velocity fields should first be POD filtered as is a common approach to allow the technique to estimate only the coherent, large scale structures. However, the sensor velocity components must be taken from the pre-filtered data set. This approach serves two purposes; firstly, in real application, the sensor measurements would not be POD filtered in real time (as doing so requires knowledge of flow over time) so it would allow the calculated $a$ matrices to be used with raw velocity sensor measurements for estimation. Secondly, a coupling between the number of POD modes chosen and the number of velocity sensors used would exist in the application of Equation 1 which would be unfavorable when using the $a$ matrix in generating real time estimations later[38].

\section{Selection of sensor locations}

The selection of sensors should take account of the limited access that would be encountered in real application in the I.C. engine. Therefore, three cases have been considered and are depicted in Figure 7: a single line of sight across the cylinder, a central cluster and a circumferential ring. For the purposes of the presented study, the proposed sensor locations are extracted from full field PIV data, the reasons for which is two-fold. Firstly, it allows some flexibility for investigative purposes: number of sensors, fraction of bore required for the line of sight condition etc. Secondly, as with this approach the full PIV vector field is known, it may be used as validation against the estimate of the vector field calculated from the sensor inputs. However, it is envisaged that alternative sensors as discussed would be used in practice - full field PIV is used here only for the investigative reasons suggested.

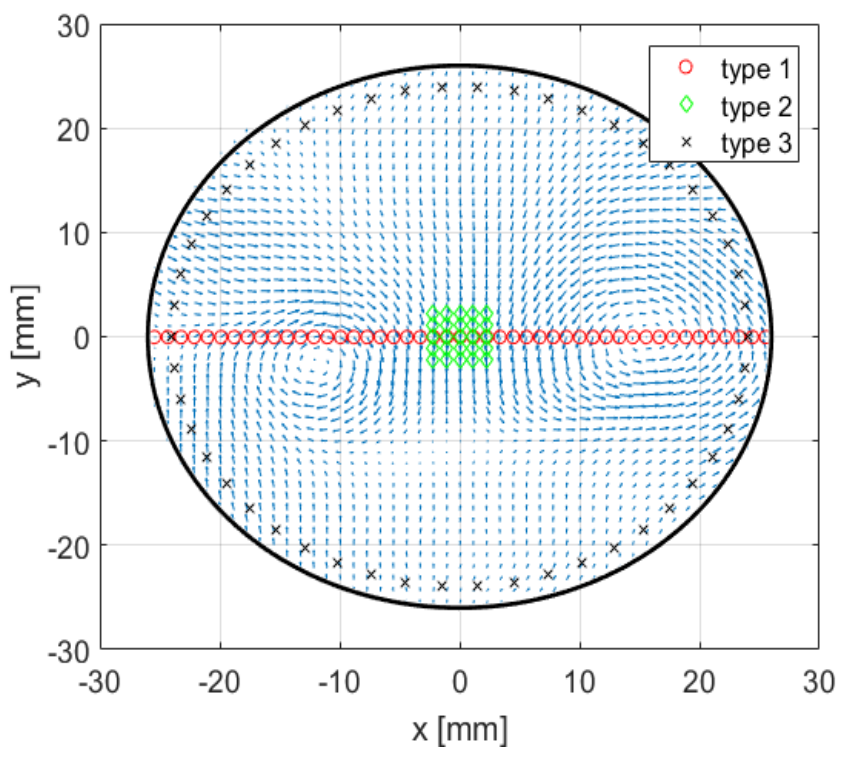

Figure 7. Depiction of three considered sensor arrangements. A typical vector field ( $100 \%$ MVL condition) is shown for context

To facilitate this approach, the total 800 velocity fields in each condition is divided into a 'slave' set of 795 which is used for the calculation of $a$ matrices. In these both the velocity at the sensor $(p)$ locations and the full velocity field $(u)$ is used with Equation 1 . The remaining 5 fields are designated 'validation' fields. From these, only the velocity vectors at the specified sensor locations $(p)$ shall be required for the estimation of these fields. As the full velocity field is already known (but not used for the calculation), it may be compared to the generated estimates as a validation exercise, described in the following section.

To provide more practical guidance to sensor system design, the number of points / areas covered by each of the suggested approaches was investigated. It could be possible that only part of the cylinder may be accessible to a practical sensing system for example. To further detail, in the case of a single, central line; points along the $y=$ $-0.03 \mathrm{~mm}$ (closest existing data to the true bisecting line) were selected as sensor measurements starting from $\mathrm{x}=-25 \mathrm{~mm}$ in steps of $1.1 \mathrm{~mm}$ (the captured PIV grid resolution), a minimum of 2 points and a maximum of 47 were used in this case.

The central cluster approach uses a minimum of $3 \times 3$ (approx. 4.8 $\mathrm{mm}^{2}$ ) around $\mathrm{x}=0, \mathrm{y}=0$. This was equally expanded for a total of 8 steps $(5 \times 5,7 \times 7, \ldots)$ up to a maximum size of $15 \times 15$ (approx. 240 $\mathrm{mm}^{2}$ ). Finally, the circumferential ring sensors were interpolated for a ring at $r=24 \mathrm{~mm}$, to ensure that there are no effects from erroneous vectors which can sometimes be found in the very extremities of the measurement plane (although were not present in the presented data set). Starting at a zero angle (i.e. $\mathrm{x}=24, \mathrm{y}=0$ ) interpolation over a constant radius was stepped by $\pi / 25$ rads to give a total of 49 points. In a similar approach to the central line case, the effect of the number of sensors was investigated with a minimum of 2 and maximum of 49. A summary of the tests carried out are detailed Table 2. The 103 tests listed, were carried out for each of the validation fields. An assessment of the estimations is provided in the following section. 
However, depending on the application and the required accuracy, the provided estimation could still be sufficient. This is further explored in the assessment of accuracy section.

\begin{tabular}{|c|l|l|l|c|}
\hline Type & Descriptor & $\begin{array}{l}\text { Minimum } \\
\text { dimension }\end{array}$ & $\begin{array}{l}\text { Maximum } \\
\text { dimension }\end{array}$ & $\begin{array}{c}\text { Total } \\
\text { estimations }\end{array}$ \\
\hline 1 & Line & $\begin{array}{l}2.2 \mathrm{~mm}(2 \\
\text { points })\end{array}$ & $\begin{array}{l}51 \mathrm{~mm}(46 \\
\text { points })\end{array}$ & 49 \\
\hline 2 & Cluster & $\begin{array}{l}4.8 \mathrm{~mm}^{2} \\
(3 \times 3 \\
\text { points })\end{array}$ & $\begin{array}{l}240 \mathrm{~mm}^{2} \\
(15 \times 15 \\
\text { points })\end{array}$ & 8 \\
\hline 3 & Ring & $\begin{array}{l}\pi / 25 \mathrm{rad}(2 \\
\text { points })\end{array}$ & $\begin{array}{l}2 \pi \mathrm{rad}(49 \\
\text { points })\end{array}$ & 46 \\
\hline & \multicolumn{3}{|r|}{ Total } & 103 \\
\hline
\end{tabular}

\section{Velocity estimation results}

As it is neither practical nor useful to present all of the velocity field estimations generated -3 valve lift conditions, 5 validation fields, 103 sensor configurations giving a total of 1545 velocity fields $-\mathrm{a}$ few are presented here to aid discussion. Following this, all the generated data will then be assessed for accuracy and a measure of representativeness is suggested allowing all tests to be compared.

\section{Sensor type 1: Line}

When using only a relatively limited number of inputs from this structured sensor set results in a poor estimation of the vector field as shown in Figure 8 which presents the case of 9 sensors.

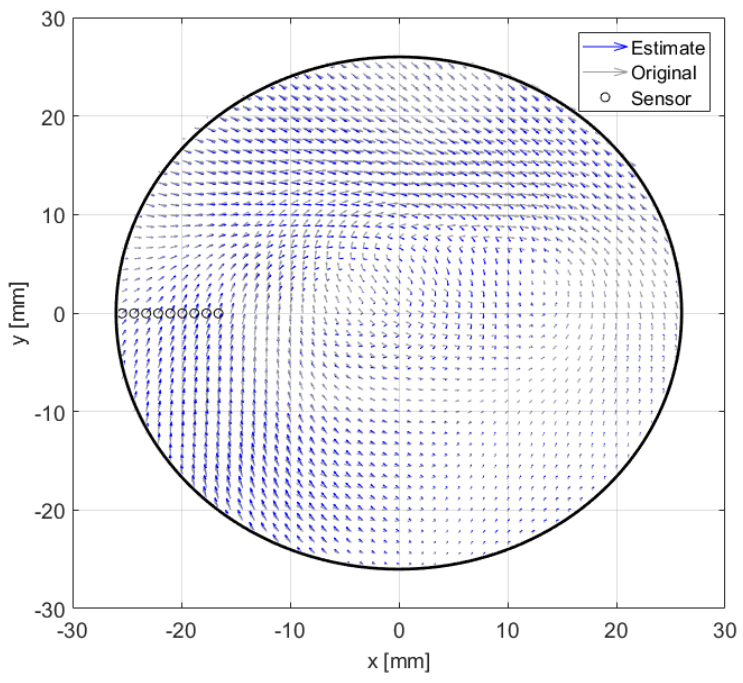

Figure 8. Estimation of velocity field using 9 type 1 sensors (vectors used as sensors marked with 'o')

However, the estimation at the points of the sensors is much closer, as one may expect when these points have themselves been used as the inputs to the estimation. Figure 9 shows how even at these points, there is a slight discrepancy between the estimate and the original field. This is due to the raw, unfiltered velocity measurements being used as sensor inputs to estimate the POD filtered velocity field. Using so few sensors has not allowed the large-scale motion to be effectively captured leading to some inaccuracies in the estimation.

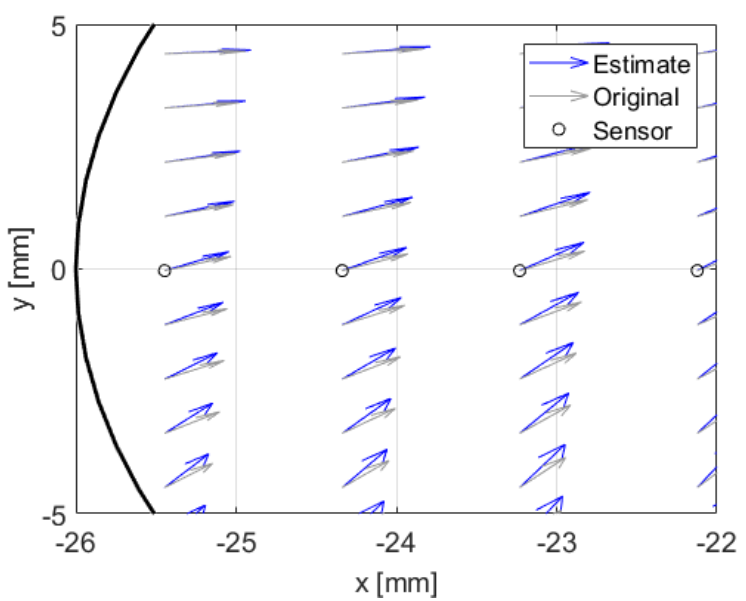

Figure 9. Extract of the estimation of velocity field using 9 type 1 sensors showing the immediate vicinity of sensors

By increasing the number of sensor points above 35 - a value suggested in literature[38] - one can see much closer agreement between the estimation and the original, shown in Figure 10. Qualitatively at least, the estimation obtained using the type 1 structured approach appears to be more representative of the original field. This may be due to the sensor covering the spatial domain in a structured, regular manner, meaning any large scale structures are more likely to be within close proximity to the sensors, and therefore have a higher spatial correlation to those areas sensed.

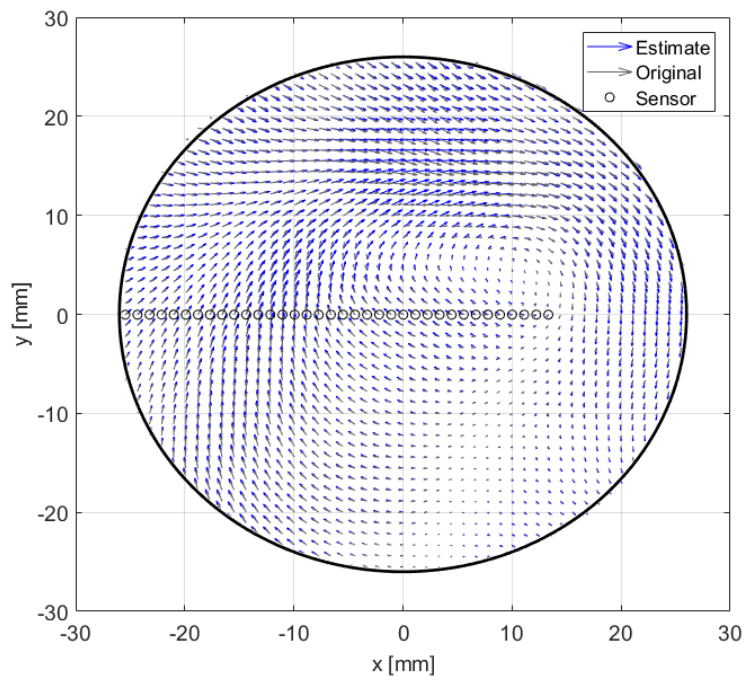

Figure 10. Estimation of velocity field using 35 type 1 sensors (vectors used as sensors marked with 'o')

\section{Sensor type 2: Central cluster}

As with the previously investigated sensor type arrangement, the illustrative example shows how the number of sensors in this arrangement effects the quality of the estimation. In this case, the first test point, $3 \times 3$ (9 points) is depicted in Figure 11. A similar observation can be made in this case, the area immediately

Page 7 of 11 
surrounding the sensor area is well estimated, with areas further away not so.

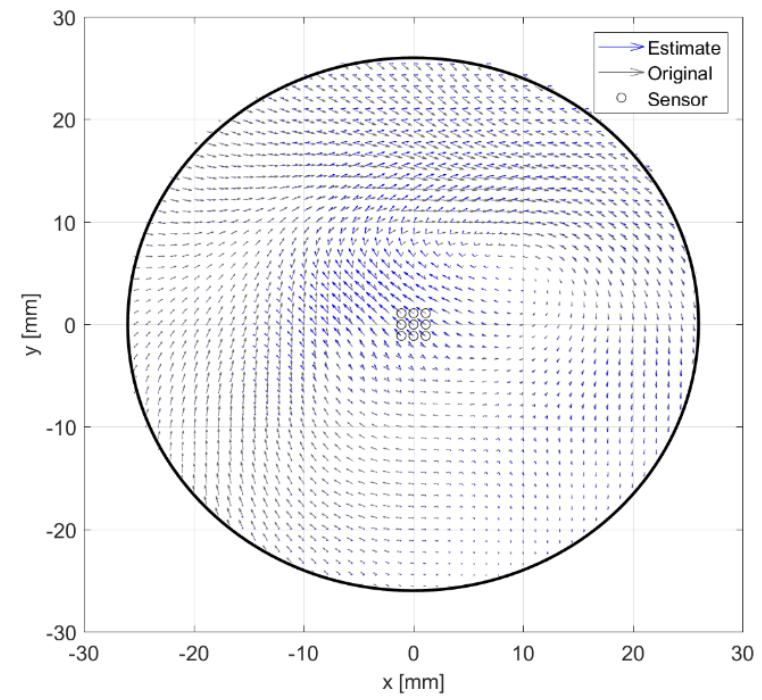

Figure 11. Estimation of velocity field using 9 ( $3 \times 3$ configuration) type 2 sensors (vectors used as sensors marked with 'o')

It can be shown in Figure 12 that the type 2 approach can provide a similar level of accuracy to the type 1 configuration but with a significantly increased sensor count; a 121 sensor configuration is depicted in Figure 12. The improvement of representation of the estimation between the 9 sensor case and 121 sensor case is highlighted in Figure 13. This presents a zoomed region of Figure 11 and 12 respectively.

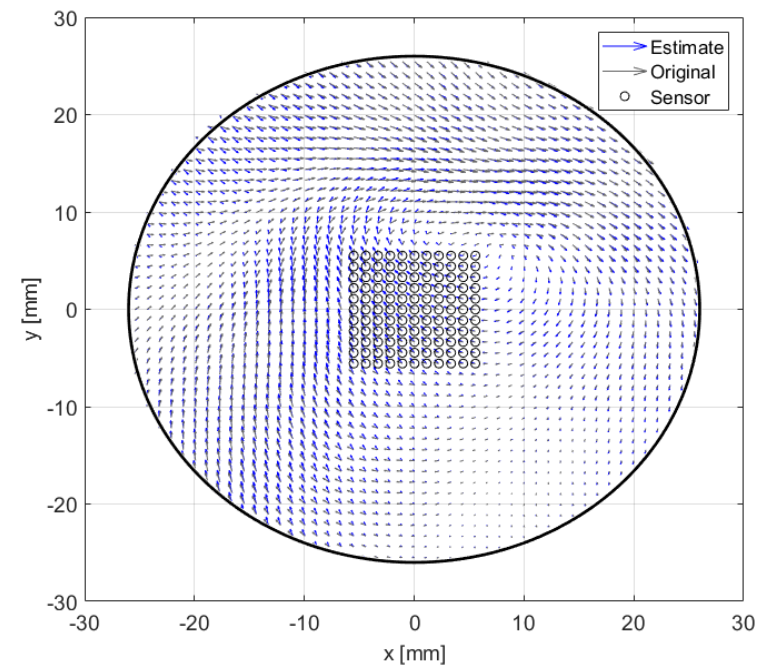

Figure 12. Estimation of velocity field using 121 (11x11 configuration) type 2 sensors (vectors used as sensors marked with 'o')

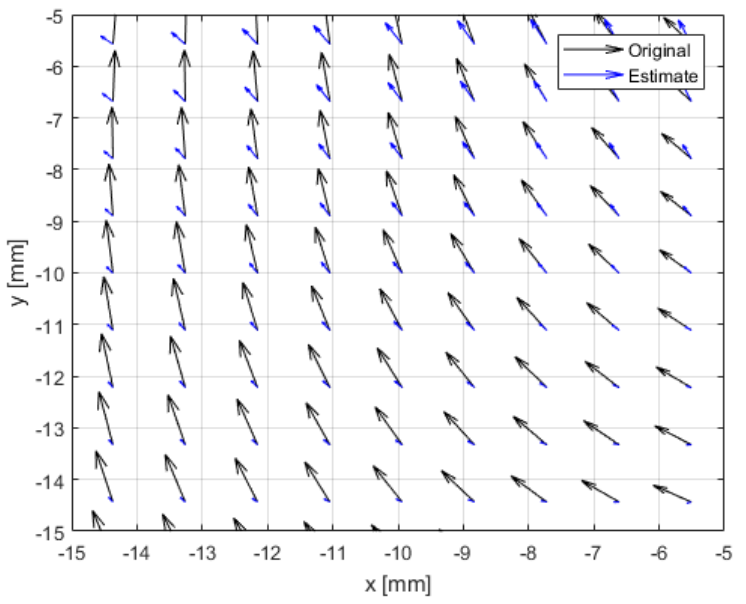

(a) Type $2-9$ sensor case

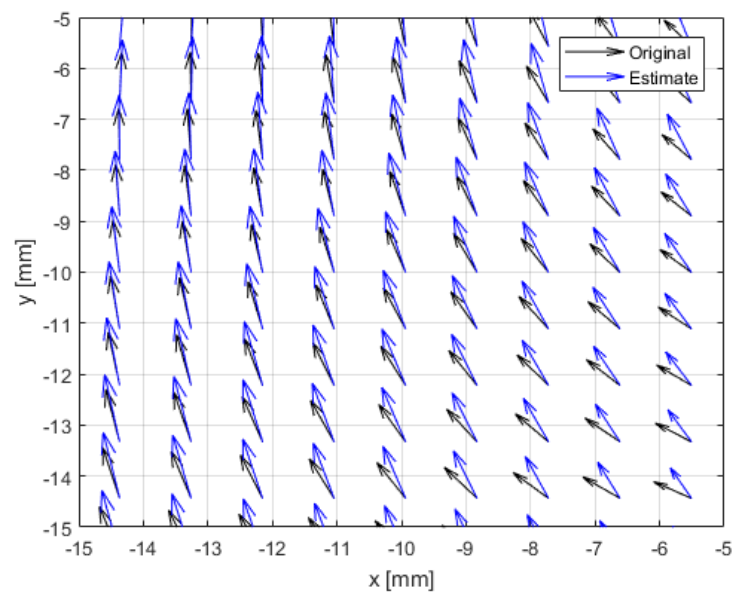

(b) Type 2-121 sensor case

Figure 13 Comparison of type 2 estimations

\section{Sensor type 3: Circumferential ring}

The final sensor configuration to be studied is the arrangement of sensors circumferentially. In contrast to the two previous sensor types, for the purposes of this study it was necessary to interpolate to generate the sensor measurements. This is due to the PIV data being aligned to a cartesian grid, and it is more practical to discuss the sensors in this section according to their angle along a constant 24 $\mathrm{mm}$ radius arc about the cylinder center with 0 rad defined as the positive $\mathrm{x}$ direction on $\mathrm{y}=0$ (i.e. the first point is located at $\mathrm{x}=24$ $\mathrm{mm}, \mathrm{y}=0 \mathrm{~mm}$ ). 


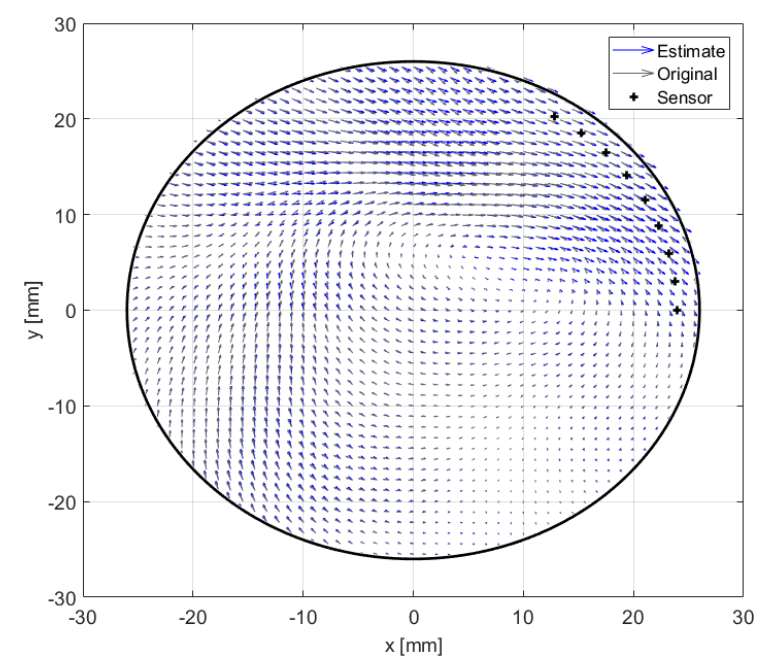

Figure 14. Estimation of velocity field using 9 type 3 sensors (interpolated sensor positions marked with '+')

In a similar fashion to the two previous configurations, Figure 14 shows that by using the type 3 configuration, vectors in the immediate vicinity of the sensors are well represented. Further increasing the number of sensors to 35, shown in Figure 15, significantly improves the representativeness of the estimation, providing a broadly accurate representation of the major flow structure.

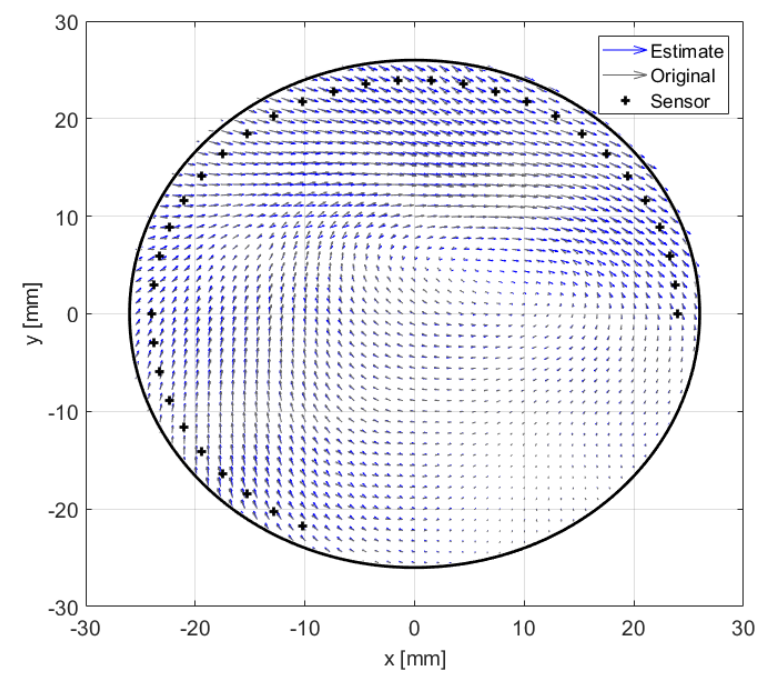

Figure 15. Estimation of velocity field using 35 type 3 sensors (interpolated sensor positions marked with '+')

\section{Assessment of accuracy}

From the data presented thus far, to differing extents, all three sensor configurations are capable of providing an accurate representation of the true flow field, albeit with different efficiencies (accuracy vs number of sensors required). Whilst Figures 9 -Figure 15 provide a visualisation of the impact of sensor configuration and number of sensors, with such a large underlying data set, it would provide more useful to calculate a measure of accuracy/representativeness of each estimation to allow the impact of sensor count to be effectively compared across the different configurations. To directly compare vector fields, one may use vector field correlation, Equation 3, where subscript 'est' denotes the estimated set and subscript 'or' denotes the original set. The resulting statistic is a measure between $0-1$ of how similar the fields are.

$R_{i i}=\frac{\overline{u_{l}(x)_{e s t} u_{l}(x)_{o r}}}{\sqrt{\overline{u_{l}^{2}(x)_{e s t}}} \sqrt{\overline{u_{l}^{2}(x)_{o r}}}}$

Correlation is carried out for each of the 103 sensor configurations reported, with the correlations for the 15 validation vector fields averaged in each case.

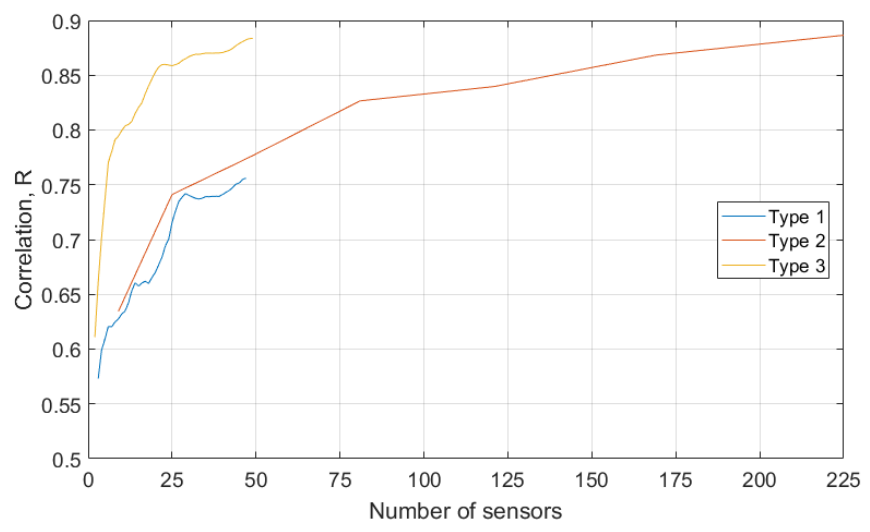

Figure 16. Summary of estimated vector field correlations with the original validation vector fields

The correlations are summarised in Figure 16. Firstly, comparing type 1 and 2 sensors, one can see that a very similar performance is achieved with this approach; with regards to correlation for a given number of sensors. This is explained by the spacing between the sensors being similar, however in the case of type 2 sensors, a higher sensor count is allowed due to expansion in both $\mathrm{x}$ and $\mathrm{y}$ dimensions. However, it would not be possible for the type 1 sensors to continue the trend of type 2 as the 49 sensor case represents full coverage of the available viewing area. Type 2 would be expected to have diminishing improvements on the correlation as sensor count is further increased. It is worth noting also that computational expense increases with the square of the number of sensors due to Equation 1, and therefore whilst the upper extremes of type 2 configuration were explored, it is likely they would not be suitable for a real-time application.

Sensor type 3 configuration sees a different trend. This is shown to more efficient than the previous two configurations. The reasons for this are two-fold. Firstly, the spacing between the sensors is larger and therefore for a given number of sensors, a larger area is covered. Secondly, as the correlation matrices are generated in a similar (note not identical) manner to spatial correlations, a greater proportion of the large structures will be in proximity to the distributed sensors in type 3 . The results for type 3 sensors are closer to those found in literature[38] where randomly distributed sensors to cover the spatial domain were used to negate effects of spatial location optimisation.

\section{Conclusions}

The work presented in this paper proposed the novel implementation of the linear stochastic estimation technique to the I.C. engine in a manner which could provide an input to a real-time active control system. It allows air motion to be estimated effectively using 
relatively few sensors which have been distributed in a manner which represents feasible, state-of-the-art velocity measurement devices. In total, three sensor configuration types were considered: a single bisecting line measurement, a central cluster and a circumferential ring. In each configuration, a sweep was performed on the number of sensors required in order to study the efficiency of each approach.

All three configurations provided estimations which correlated well with the validation vector field data, but with key differences between them. Firstly, both type 1 and 2 were found to perform with a similar efficiency (that is the number of sensors required for an expected level of correlation) due in part to their similar sensor spacing and proximity area coverage. However, by arranging the sensors in a ring around the edge of the cylinder, as in type 3 , a greater level of correlation was achieved.

For each sensor configuration the LSE approach performed equally well under all three MVL strategies without need for calibration to individual cases, showing that the technique could be applied to a wide range of expected operating conditions.

Ultimately, sensor location will likely be dictated by available access to the I.C. engine and the presented work shows that even with suboptimal distribution or arrangement of sensors, the LSE approach can be a useful tool for future active engine control systems. The number of sensors required could be achievable with current optical based sensor techniques.

In order to realize intra-cycle control of IC engines it is necessary to characterize the flow field in any given cycle. Previous work by the authors [38] has indicated that combined use of POD and LSE along with some limited randomly located sensor information from a given cycle can be successfully used to estimate the current cycle incylinder flow field. This work looks at clustering the sensor locations into configurations that although practical may lead to degradation of the LSE accuracy. The findings above indicate that the configurations considered do allow LSE to be used and has allowed the configurations to be ranked. Future work is now directed at designing a suitable sensor for providing input to the LSE approach that may enable the opportunities that intra-cycle control may offer.

\section{References}

1. Braisher, M., Stone, R., and Price, P., "Particle Number Emissions from a Range of European Vehicles," SAE Tech. Pap. 2010-1-7, 2010, doi:10.4271/2010-01-0786.

2. Johansson, A.N., Hemdal, S., and Dahlander, P., "Experimental Investigation of Soot in a Spray-Guided Single Cylinder GDI Engine Operating in a Stratified Mode," 2013-24-00, 2013, doi:10.4271/2013-24-0052.

3. Leach, F., Knorsch, T., Laidig, C., Wiese, W., and Bosch, R., “A Review of the Requirements for Injection Systems and the Effects of Fuel Quality on Particulate Emissions from GDI Engines," SAE Tech. Pap. 2018-01-17, 2018, doi:10.4271/2018-01-1710.Abstract.

4. Raza, M., Chen, L., Leach, F., and Ding, S., "A Review of Particulate Number (PN) Emissions from Gasoline Direct Injection (GDI) Engines and Their Control Techniques," Energies 11(6), 2018, doi:10.3390/en11061417.

5. Sharma, N. and Agarwal, A.K., "Effect of the Fuel Injection Pressure on Particulate Emissions from a Gasohol (E15 and M15)Fueled Gasoline Direct Injection Engine," Energy \& Fuels 31(4):4155-4164, 2017, doi:10.1021/acs.energyfuels.6b02877.

Page 10 of 11
6. Baby, X., Dupont, A., Ahmed, A., Sa, R., Deslandes, W., and Charnay, G., "A New Methodology to Analyze Cycle-to-Cycle Aerodynamic Variations," SAE Tech. Pap. (2002-01-2837), 2002, doi:10.4271/2002-01-2837.

Teh, K., Ge, P., Wang, Y., and Hung, D., "Cycle-to-Cycle Variation of In-Cylinder Tumble Flow by Moment Normalization," 2018, doi:10.4271/2017-01-2214.Copyright.

8. Stone, C.R., Brown, a G., and Beckwith, P., "Cycle-by-Cycle Variations in Spark Ignition Engine Combustion - Part II: Modelling of Flame Kernel Displacements as a Cause of Cycle-by-Cycle Variations," SAE Tech. Pap. (412), 1996, doi:10.4271/960613.

9. Brown, a G., Stone, C.R., and Beckwith, P., "Cycle-by-Cycle Variations in Spark Ignition Engine Combustion - Part I: Flame Speed and Combustion Measurements and a Simplified Turbulent Combustion Model," SAE Tech. Pap. (412), 1996, doi:10.4271/960612.

10. Xingcai, L., Libin, J., Junjun, M., and Zhen, H., "Experimental study on the cycle-by-cycle variations of homogeneous charge compression ignition combustion using primary reference fuels and their mixtures," Proc. Inst. Mech. Eng. Part D J. Automob. Eng. 221(7):859-866, 2007, doi:10.1243/09544070JAUTO481.

11. Sun, S., Eilts, P., Scholz, P., and Haubold, S., "Active Control of Cylinder Charge Motion Using Vortex Generating Jets (VGJs) on Generic Intake Port Geometries," SAE Int. J. Engines 11(4):03-1104-0032, 2018, doi:10.4271/03-11-04-0032.

12. Butcher, D., Spencer, A., and Chen, R., "Influence of asymmetric valve strategy on large-scale and turbulent in-cylinder flows," Int. $J$. Engine Res. 19(6):631-642, 2018, doi:10.1177/1468087417725232.

13. Adrian, R.J., "On the Role of Conditional Averages in Turbulence Theory,” Turbul. Liq. (JANUARY 1977):323-332, 1977.

14. Adrian, R. and Moin, P., "Stochastic estimation of organized turbulent structure: homogeneous shear flow," J. Fluid Mech. 190(1988):531-559, 1988, doi:10.1017/S0022112088001442.

15. Cole, D., Glauser, M., and Guezennec, Y., "An Application of the Stochastic Estimation to the Jet Mixing Layer," Phys. Fluids 4(1):192-194, 1991.

16. Druault, P., Yu, M., and Sagaut, P., "Quadratic stochastic estimation of far-field acoustic pressure with coherent structure events in a 2D compressible plane mixing layer," Int. J. Numer. Methods Fluids 62(8):906-926, 2010, doi:10.1002/fld.2047.

17. Lumley, J.L., "The Structure of Inhomogeneous Turbulent Flows," Atmos. Turbul. Radio Wave, Nauk. 166-178, 1967.

18. Cosadia, I., Borée, J., Charnay, G., and Dumont, P., "Cyclic variations of the swirling flow in a Diesel transparent engine," Exp. Fluids 41(1):115-134, 2006, doi:10.1007/s00348-006-0163-4.

19. Cosadia, I., Borée, J., and Dumont, P., "Coupling time-resolved PIV flow-fields and phase-invariant proper orthogonal decomposition for the description of the parameters space in a transparent Diesel engine," Exp. Fluids 43(2-3):357-370, 2007, doi:10.1007/s00348007-0338-7.

20. Chen, H., Reuss, D.L., and Sick, V., "Analysis of misfires in a direct injection engine using proper orthogonal decomposition," Exp. Fluids 51(4):1139-1151, 2011, doi:10.1007/s00348-011-1133-z.

21. Ge, P. and Hung, D.L.S., "Investigation of Cycle-to-Cycle Variation of In-Cylinder Engine Swirl Flow Fields Using Quadruple Proper Orthogonal Decomposition," J. Eng. Gas Turbines Power 139(7):072803, 2017, doi:10.1115/1.4035628. 
Druault, P., Guibert, P., and Alizon, F., "Use of proper orthogonal decomposition for time interpolation from PIV data: Application to the cycle-to-cycle variation analysis of in-cylinder engine flows," Exp. Fluids 39(6):1009-1023, 2005, doi:10.1007/s00348-005-0035-

23. Voisine, M., Thomas, L., Borée, J., and Rey, P., "Spatio-temporal structure and cycle to cycle variations of an in-cylinder tumbling flow," Exp. Fluids 50(5):1393-1407, 2011, doi:10.1007/s00348010-0997-7.

24.

Hosseini, Z., Martinuzzi, R.J., and Noack, B.R., "Sensor-based estimation of the velocity in the wake of a low-aspect-ratio pyramid," Exp. Fluids 56(1), 2015, doi:10.1007/s00348-014-18808 .

25. Taylor, J.A. and Glauser, M.N., "Towards practical flow sensing and control via POD and LSE based low-dimensional tools," $J$ Fluids Eng. 126(3):337-345, 2004, doi:Doi 10.1115/1.1760540.

Gutmark, E.J., Verfaillie, S., Bonnet, J.-P., and Grinstein, F., "Linear Stochastic Estimation of a Swirling Jet," AIAA J. 44(3):457-468, 2006, doi:10.2514/1.19237.

27. Arnault, A., Dandois, J., Monnier, J.C., Delva, J., and Foucaut, J.M. "Analysis of the filtering effect of the stochastic estimation and accuracy improvement by sensor location optimization," Exp. Fluids 57(12):1-22, 2016, doi:10.1007/s00348-016-2276-8.

28. Muradore, R., Bezzo, F., and Barolo, M., "Optimal sensor location for distributed-sensor systems using multivariate regression," Comput. Chem. Eng. 30(3):521-534, 2006, doi:10.1016/j.compchemeng.2005.10.009.

29. Cohen, K., Siegel, S., and McLaughlin, T., "A heuristic approach to effective sensor placement for modeling of a cylinder wake," Comput. Fluids 35(1):103-120, 2006, doi:10.1016/j.compfluid.2004.11.002.

Kerhervé, F., Roux, S., and Mathis, R., "Combining time-resolved multi-point and spatially-resolved measurements for the recovering of very-large-scale motions in high Reynolds number turbulent boundary layer," Exp. Therm. Fluid Sci. 82:102-115, 2017, doi:10.1016/j.expthermflusci.2016.10.032.

31. Schneiders, J.F.G., Scarano, F., Jux, C., and Sciacchitano, A., "Coaxial volumetric velocimetry," Meas. Sci. Technol. 29(6), 2018, doi:10.1088/1361-6501/aab07d.

32. Stansfield, P., Wigley, G., Catto, J., and Pitcher, G., "PIV Analysis of In-Cylinder Flow Structures over a Range of Realistic Engine Speeds," 13th Int Symp on Applications of Laser Techniques to Fluid Mechanics, Lisbon, 2006.

33. Melling, A., "Tracer particles and seeding for particle image velocimetry," Meas. Sci. Technol. 8:1406-1416, 1997.

34. Adrian, R.J. and Westerweel, J., "Particle Image Velocimetry," Cambridge University Press, 2011.

35. Stone, R., "Introduction to Internal Combustion Engines," 4th ed., Palgrave Macmillian, London, 2012.

36. Lancaster, D.R., "Effects of Engine Variables on Turbulence in a Spark-Ignition Engine,” SAE Tech. Pap. (760159), 1976, doi: $10.4271 / 760159$.

37 .

Li, Y., Zhao, H., and Ladommatos, N., "Analysis of large-scale flow characteristics in a four-valve spark ignition engine," Proc. Inst. Mech. Eng. Part D J. Automob. Eng. 216(June):923-938, 2002.

38.

Butcher, D. and Spencer, A., "Linear stochastic estimation of coherent structures in I.C. engines flow," Int. J. Engine Res., 2019.

\section{Contact Information}

Daniel Butcher

Department of Aeronautical \& Automotive Engineering, Loughborough University, Loughborough, UK

D.Butcher@lboro.ac.uk

Adrian Spencer

Department of Aeronautical \& Automotive Engineering, Loughborough University, Loughborough, UK

A.Spencer@lboro.ac.uk

\section{Acknowledgments}

The authors gratefully acknowledge the support of Group Lotus for providing use of the optical research engine, SCORE and AVT system used for the experiment part of the work presented.

\section{Definitions/Abbreviations}

AVT

CAD

CCV

GDI

MVL

LSE

PFI

PIV

POD

SCORE

VVT
Active valve train (Lotus

Engineering)

Crank angle degree

Cycle-to-cycle variation

Gasoline direct injection

Maximum valve lift

Linear stochastic estimation

Port fuel injection

Particle image velocimetry

Proper orthogonal decomposition

Single cylinder optical research system (Lotus Engineering)

Variable valve timing

Page 11 of 11

$10 / 19 / 2016$ 